\title{
Alfred Testard de Marans, Souvenirs des îles Marquises, Groupe Sud-Est, 1887-1888
}

Publications de la Société des Océanistes 48, Paris, 2004

\section{Gilles Bounoure}

\section{OpenEdition}

\section{Journals}

Édition électronique

URL : http://journals.openedition.org/jso/1045

DOI : $10.4000 /$ jso. 1045

ISSN : $1760-7256$

\section{Éditeur}

Société des océanistes

Édition imprimée

Date de publication : 1 décembre 2007

Pagination : 329-330

ISBN : 978-2-85430-010-9

ISSN : 0300-953x

\section{Référence électronique}

Gilles Bounoure, "Alfred Testard de Marans, Souvenirs des îles Marquises, Groupe Sud-Est, 1887-1888 », Journal de la Société des Océanistes [En ligne], 125 | Année 2007-2, mis en ligne le 23 avril 2008, consulté le 24 septembre 2020. URL : http://journals.openedition.org/jso/1045 ; DOI : https://doi.org/ $10.4000 /$ jso. 1045 


\section{COMPTES RENDUS}

Alfred Testard de Marans, 2004. Souvenirs des îles Marquises, Groupe Sud-Est, 1887-1888, Paris, Publications de la Société des Océanistes 48, XIV-212 p., avantpropos de la Dominique Cadilhac, avertissement de Michel Orliac, notes, glossaire, index des noms de personnes et de lieux par Marie Noëlle OttinoGaranger, bibliographie, dix figures dans le texte, douze planches hors-texte.

« Contribuer à faire connaître ces îles lointaines où s'étend notre suprématie », telle était l'ambition avouée d'Alfred Testard de Marans (1860-1890) en rédigeant ses Souvenirs des îles Marquises, datés de « février-juin 1889 », tandis qu'il dirigeait un service de l'administration coloniale au Sénégal. Où les aurait-il publiés, si les « fièvres tropicales » ne l'avaient emporté un peu plus d'un an après, en laissant ces feuillets inédits, heureusement retrouvés et reproduits dans le présent ouvrage ? Pour le ton et l'intention, ils procèdent en tout cas du genre alors florissant, en France comme dans d'autres pays d'Europe, dans les journaux comme chez les éditeurs, des « réflexions sur la question coloniale », l'accroissement des « empires » occidentaux ayant apparemment besoin de ce soutien éditorial. Ses « conclusions » sont des plus conformes à cette perspective, elles visent à « faciliter le développement économique de ces îles », mais par-dessus tout, à « faciliter dans ces contrées la colonisation et le développement de notre commerce national ».

Par chance, l'aide-commissaire de la Marine versé dans « la Coloniale » Testard de Marans, en assumant la charge de sous-administrateur des Marquises pour le groupe Sud-Est d'octobre 1887 à avril 1888, peutêtre par ambition, ou pour sortir du «trantran » du service du Détail des subsistances qu'il dirigeait auparavant à Papeete, se prit d'un intérêt profond pour la nouvelle tâche et les «administrés » qui lui étaient pour la première fois confiés. Où et quand eut-il la possibilité de se documenter, pour rédiger son « histoire » de la découverte et de la colonisation des Marquises (premier chapitre, 24 pages), étonnamment fournie et «à jour », jusqu'à se référer au livre de Deschanel paru en 1888, Les Intérêts français dans l'océan Pacifique ? Les brèves informations biographiques livrées par la « $4^{\mathrm{e}}$ de couverture » (cette personnalité mériterait une étude approfondie) ne laissent conjecturer, entre ses affectations successives, aucun temps libre qu'il ait pu consacrer à de telles recherches.
Un fragment de lettre à sa famille (reproduit pl. V), cocassement écrite depuis «Atuona, capitale des cannibales, etc. ce 10 février $1888 »$, indique comme il était déjà frotté de culture polynésienne, jusqu'à gloser sur le même ton cette expression tahitienne: "Tirara parao [parau], fini de blaguer... ».

À près de cent-dix ans de distance, l'ouvrage conçu par Testard de Marans en vue d'améliorer le sort des colons comme des Marquisiens laisse forcément au lecteur des sentiments mêlés. À côté d'une perspective « impériale » indépassable pour l'auteur et de probables querelles de prérogatives administratives pour le contrôle d'un archipel alors subordonné à Papeete et dont il prône « l'autonomie financière [...] sous l'autorité complète du Résident », le texte révèle une réelle sympathie pour les insulaires et leur pays, allant jusqu'aux observations naïves du " voyageur » soulignant le « pittoresque » des situations et des paysages (p. 106). Sa sincérité à transcrire les informations dont il avait connaissance semble confirmée par des témoins indépendants. Par exemple le yachtsman britannique James Cumming Dewar, qui fit escale à Fatu Hiva et Hiva Oa dans les derniers jours d'août 1888, et y rencontra Fanny et Robert Louis Stevenson (Voyage of the Nyanza... being the record of a three years' cruise in a schooner yacht..., Édimbourg-Londres, W. Blackwood and Sons, 1892 : 170), remarquait :

«I was informed that frequently when the priest came over he was unable to get a single person to attend the service. As a matter of fact, the natives are little changed from their condition when the French first landed [...] cannibalism has virtually disappeared, though I heard that a man had been eaten only two years ago at Hiva-oa. »

\section{Testard de Marans n'en juge pas autrement :}

« Le protestantisme semble avoir fait bien moins de prosélytes que la religion catholique, à laquelle les Marquisiens appartiennent presque tous au moins en théorie, car en pratique ils sont, à peu d'exceptions près, fort indifférents. La conversion des indigènes est plutôt apparente que réelle. » (p. 70)

«L'anthropophagie peut être considérée comme disparue du groupe Sud-Est à l'heure actuelle. Le dernier Canaque mangé, un nommé Matupa, l'a été sur le plateau des Hamau en 1887. » (p. 179)

Reste à alerter les lecteurs sur la part réduite qu'occupent souvent les « souvenirs » dans cette description des îles Marquises plus proche des «statisti- 
ques monumentales » du milieu du XIX ${ }^{\mathrm{e}}$ siècle que des récits de voyage de la même époque. Au fil du très précieux travail d'annotation, d'élucidation et de commentaire dont elle a enrichi ce texte, et qui suffirait à lui seul à rendre ce volume indispensable aux chercheurs, Marie Noëlle Ottino-Garanger indique à diverses reprises que Testard de Marans puise dans ses lectures soit des citations dont il n'indique pas la source, soit des développements dont il s'attribue la paternité. D'autres emprunts plus ou moins furtifs valent d'être signalés, par exemple la longue citation sans source des pp. 11-16, reproduisant les pp. 126-137 des Derniers Sauvages (Paris, Hachette, 1860 et version antérieure de la Revue des Deux-Mondes) de Max Radiguet, ou encore le chapitre dévolu aux " mœurs et coutumes », où l'auteur des Souvenirs a démarqué (p. 122), en l'amplifiant, un paragraphe sur «l'origine des Marquésans » qu'il avait lu dans l'ouvrage d'Henri Le Chartier, Tahiti et les colonies françaises de la Polynésie, (Paris, Jouvet et Cie, 1887 : 197, parmi de nombreux autres emprunts), avant d'évoquer les vehine hae ou « revenants » (pp. 130-131) en des termes souvent repris de l'ouvrage alors tout récent d'Aylic Marin [Édouard Petit, futur gouverneur des Établissements Français d'Océanie], En Océanie (Bayle, Paris, 1888 : 97-112).

En somme, plus on découvre en Testard de Marans un lecteur et un compilateur de premier ordre, moins il s'avère facile de mesurer et de reconnaître ses talents d'observateur: ses Souvenirs livrent certainement beaucoup de ce qu'on pouvait savoir des Marquises en 1888 (et qui n'est pas nécessairement inexact pour être de deuxième main, mais demande vérification), moins nettement ce que pouvait voir sur place le visiteur, en fonction de sa perspicacité, de sa liberté ou de ses préventions. Maintenant qu'ils disposent grâce à la Société des Océanistes de ce texte inédit, remarquablement servi par une édition soigneusement établie et très judicieusement illustrée de documents d'époque, c'est aux historiens et aux chercheurs de déterminer en détail ce qu'a vu vraiment Testard de Marans aux Marquises, et de quel prix est son témoignage. D'un tel passage au crible, qu'on ne saurait limiter à ce seul texte, car, par un trait d'époque, nombre de ses sources résultent elles-mêmes de compilations, on pourrait sans doute attendre un tableau plus précis et authentique des îles Marquises à la fin du XIX ${ }^{\mathrm{e}}$ siècle.

\section{Gilles BOUNOURE}

Joseph de Rosamel, 2005. Pohnpei. Micronésie en 1840. Voyage de circumnavigation de la Danaïde, édité par Pierre de Rosamel et Jean-Christophe Galipaud, Paris, Publications de la Société des Océanistes 49, 160 p., bibliographie, index, cartes, ill. noir et blanc.

Les témoignages occidentaux sur la Micronésie traditionnelle sont tous de grand prix, du fait même de leur rareté : on ne compte guère qu'une vingtaine de textes en langue française, au xixe siècle, traitant un peu précisément de ce qu'on nommait alors les îles Carolines, possédées ou revendiquées nominalement par une Espagne alors au bord de l'implosion. Mais pour la plupart hors de toute perspective «scientifique ", ou au moins de libre examen. Les négociations du traité de Berlin, en 1885, ont ainsi donné lieu à des publications visant à dénoncer l'impérialisme germanique... au profit d'autres, la « ligne bleue des Vosges » trouvant ici à se prolonger dans les reflets mouvants du Pacifique. Par exemple, dans Les Îles Carolines, étude générale, Paris, Chalamel Aîné, Librairie coloniale, 1885, 64 pp. , ill. et carte, Adolphe Gouts, Secrétaire de la rédaction de la Science Universelle, arrivait à cette conclusion, p. 63 :

«Plusieurs États européens revendiquent en ce moment la possession des Carolines. Notre partie historique, rédigée avec la plus grande impartialité, d'après des documents absolument irréfutables, montre de quel côté est le bon droit. »

Son introduction précisait d'avance (p. 12) :

«On a pu voir par ce qui précède que la prétention de l'Allemagne est inique. »

Le texte inédit ici publié par la Société des Océanistes échappe-t-il à cette perspective colonialiste animant la plupart des observateurs du $\mathrm{XIX}^{\mathrm{e}}$ siècle, et souvent les meilleurs ? Ce volume le laisse croire. Dans sa « note liminaire », Jean-Christophe Galipaud indique que l'auteur, le capitaine de vaisseau Joseph de Rosamel (1807-1853), qui participa comme enseigne à l'expédition d'Alger en 1830, « est le second fils du vice-amiral Claude de Rosamel, ministre de la Marine de 1836 à 1839, qui organisa la reprise des grands voyages scientifiques d'exploration confiés à Dumont d'Urville, Dupetit-Thouars et Laplace» (p. 7). Dans son « autoportrait sous forme d'une lettre apocryphe adressée par l'auteur à Pierre de Rosamel », ce descendant du narrateur, "inventeur » de ce texte qu'il a retrouvé dans les archives familiales, assure que les instructions données par Louis-Philippe à son aïeul envoyé en mission dans le Pacifique ne tendaient qu'à cette conclusion :

«Dans tous les lieux où se montrera la corvette la Danaïde, vous mettrez vos soins à lier avec les autorités et les habitants des relations amicales et à donner une idée avantageuse de la puissance de la France. » (p. 22)

À suivre l'une et l'autre de ces présentations, en envoyant dans le Pacifique le "man of war" la Danaïde, Louis-Philippe et son ministre de la Marine n'auraient fait que préfigurer au moins en esprit les missions à la fois " amicales » et «scientifiques" aujourd'hui confiées par les autorités de ce pays-ci à des institutions comme l'Alliance Française ou l'IRD, qui a contribué à l'édition de ce volume.

Il n'est pas certain que les Tahitiens, Mangaréviens et Marquisiens instruits du processus de colonisation de leurs îles soient aujourd'hui disposés à admettre que Dupetit-Thouars, Dumont d'Urville et autres officiers supérieurs de la «Royale » avaient été dépêchés dans le Pacifique pour de simples « voyages scientifiques d'exploration », à la même époque où les marins français cherchaient aussi à planter le drapeau tricolore en Nouvelle-Zélande, ou, par missionnaires 